\title{
Einbindung von vielen Teilnehmenden in den softwaregestützten Ideenfindungsprozess
}

\author{
Marcel Brandt ${ }^{1} \cdot$ Philipp Bader · Navid Tavanapour ${ }^{2}$ \\ Angenommen: 13. April 2021 / Online publiziert: 18. Mai 2021 \\ (c) Der/die Autor(en) 2021
}

\section{Zusammenfassung}

In diesem Artikel werden die Entwicklung und Evaluierung eines Ideenfindungsprozesses, der über eine hohe Anzahl von Teilnehmenden skaliert werden kann, vorgestellt. Ziel dieser Untersuchung ist, viele Personen effizient in diesen Prozess einzubinden. Das zentrale Problem bei der Erhöhung der Anzahl der Teilnehmenden liegt darin, dass nicht ohne Weiteres große Personengruppen in einen herkömmlichen Ideenfindungsprozess einbezogen werden können, weil sie sich nicht effizient beteiligen können. Es wird hierbei untersucht, wie gleichzeitig und am gleichen räumlichen Ort Ideen digital gesammelt, kategorisiert, priorisiert und vorgestellt werden können. Dafür wurde ein Prozess auf der Grundlage der 1-2-4-All-Methode und eine Softwarelösung mit dem Konzept der Evolution-Methode konzipiert, prototypisch umgesetzt und evaluiert.

\section{Einleitung}

Innovation gilt als wichtiger Wettbewerbsvorteil für Unternehmen [16, S. 322, 6, S. 1, 24, S. 2f.]. Um dauerhafte Innovationen zu gewährleisten, müssen in Unternehmen kontinuierlich Ideen gefunden und bewertet werden [6, S. 1]. Moderatoren können im Rahmen eines Präsenzmeetings mit vielen Teilnehmenden, anhand von Kreativitätsmethoden, Ideen sammeln. Gleichzeitig wird durch die Einbindung vieler Teilnehmenden eine für die Umsetzung notwendige Akzeptanz erzeugt.

Als Beispiel für eine Anwendung kann hier das Infrastrukturprojekt „Stuttgart 21“ für schwerwiegende Folgekosten aufgrund nicht eingebundener Stakeholder gesehen werden [1]. Durch eine frühzeitige Einbindung der Bür-

Marcel Brandt

marcel.brandt@iteratec.com

Philipp Bader

ph.bader@gmx.de

$\triangle$ Navid Tavanapour

tavanapour@informatik.uni-hamburg.de

1 iteratec GmbH, Am Sandtorkai 75, 20457 Hamburg, Deutschland

2 Fakultät für Mathematik, Informatik und Naturwissenschaften Informatik, Universität Hamburg, Vogt-Kölln-Straße 30, 22527 Hamburg, Deutschland ger/innen, wie beispielsweise bei dem erfolgreichen Projekt der Y-Trasse [5], hätten diese zusätzlichen Aufwände sowie Proteste vermieden oder mindestens verringert werden können. Durch die Einbindung wird eine intrinsisch erzeugte Motivation geschaffen [13, S. $1122 \mathrm{ff} ., 27$, S. 15], sodass die Unternehmensziele zu den Zielen der Mitarbeiter/innen werden, sie beschleunigt umgesetzt oder sowie bei Stuttgart 21 erst ermöglicht werden [24, S. 38, 1]. In Projekten und politischen Entscheidungen müssen immer häufiger viele Personen eingebunden werden, damit große Vorhaben nicht an den Betroffenen scheitern [1, 5, 24, S. 23].

iteratec hat im Jahr 2019 die Genossenschaft nurdemteam eG mit über 200 Mitgliedern gegründet. Im Rahmen dieser Genossenschaft wurde dieses Vorhaben, bei dem viele Personen involviert sein müssen, untersucht. Durch verschiedene Projekte kann die nurdemteam eG bei Problemen vorbeugen, indem sie die große Anzahl an Mitarbeitenden von Anfang an in die Ideenfindung einbindet.

Es stellt sich daher die Frage, wie eine derartige Einbindung von vielen Personen geschehen kann. Diese Einbindung ist lohnend, weil bekannt ist, dass beim Brainstorming durch die Quantität der Ideen auch die Anzahl der qualitativ hochwertigen Ideen zunimmt [17, 18, S. 38].

Als mögliche Lösung für die Einbindung von vielen Teilnehmenden kann die 1-2-4-All-Kreativmethode genutzt werden. Hierbei werden Ideen von Gruppen mit steigender Personenanzahl $(1,2,4$ oder alle) in jedem Schritt vorgestellt und überarbeitet. 
Das zentrale Problem bei der Erhöhung der Anzahl der Teilnehmenden ist, dass nicht ohne Weiteres eine große Personenmenge in einen herkömmlichen Ideenfindungsprozess einbezogen werden kann [28, S. 35]. Ein weiteres Problem stellt die Moderation eines Workshops mit vielen Teilnehmenden dar [28, S. 35]. Zusätzlich ist die Interaktion zwischen den Teilnehmenden gehemmt, wodurch eine Verschwendung von kognitiven Ressourcen entstehen kann [28, S. 35], welche im Unternehmen für einen ineffizienten Einsatz von Personal und deren Kosten steht. Das Problem der Verschwendung der Ressourcen wird darüber hinaus verstärkt, dass bei Mehrfachnennung der gleichen Idee eine Auswahl der Top-Ideen schwerer fällt. Daher resultiert aus den Schwierigkeiten folgende Forschungsfrage (FF) für die Umsetzung:

FF1: ,Wie kann auf der Grundlage der 1-2-4-All-Methode ein Ideenfindungsprozess erstellt werden, der die digitale Ideenfindung im skalierten Teilnehmerfeld unterstützt?“

Um FF1 zu adressieren, wurden im ersten Schritt Experteninterviews nach Meuser und Nagel [15] durchgeführt. Auf Basis der Experteninterviews wurde das Problem der Skalierbarkeit im 4. Schritt „All“ der 1-2-4-All-Methode erkannt. Bei einer großen Anzahl von Teilnehmenden sind im 4. Schritt 1-2-4-All zu viele Ideen vorhanden, um alle Ideen zeitlich effizient vorzustellen. Daher wird der Prozess wie von den Expert/innen empfohlen zwischen dem 3. und 4. Schritt durch eine Kategorisierung und Bewertung der Ideen erweitert. Mit dieser Auswahl der Ideen durch die Teilnehmenden, können dann im 4. Schritt „All“ ausgewählte Ideen vorgestellt werden.

Das Ziel ist, allen Teilnehmenden eine digitale manuelle und zeitgleiche Kategorisierung und Bewertung von Ideen im Zwischenschritt anzubieten. Dies führt zu FF2: „Wie kann das manuelle Kategorisieren im Ideenfindungsprozess durch Software unterstützt werden?"

Um diese Forschungsfragen $\mathrm{zu}$ eruieren, werden $\mathrm{zu}-$ nächst die genutzten Methoden im Grundlagenabschnitt aufgezeigt. Im Anschluss wird die Methodik für das Forschungsvorhaben dargelegt. Daraufhin folgt die Beschreibung der Durchführung der oben genannten Experteninterviews. Mit den daraus gewonnen Anforderungen wird der Ideenfindungsprozess entwickelt. Anschließend wird der Prozess im ersten Workshop validiert. Aus den Erkenntnissen resultiert die Notwendigkeit einer prototypischen Softwareentwicklung. Der umgesetzte Prototyp wird in Workshop 2 untersucht.

\section{Grundlagen}

Zunächst werden die Grundlagen dargelegt, auf denen die Inhalte des Artikels aufbauen. Dazu werden die Einord- nung im Innovationsmanagement und die Methoden für die Zusammenarbeit vorgestellt.

Die Ideenfindung ist ein Teil des Innovationsmanagements, welches alle Schritte des Innovationsprozesses umfasst [7, S. 15]. Der Innovationsprozess erstreckt sich von der ersten Idee, über das Ausarbeiten bis zur Einführung des Produktes/Services [7, S. 15]. Eine Idee ist erst nach der Entwicklung und erfolgreichen Einführung als Innovation zu betrachten [7, S. 15, 25, S. 369].

In diesem Artikel geht es zentral um die gemeinsame Ideenfindung, -bewertung und -auswahl im skalierten Personenfeld. Bei der Ideenfindung werden Ideen zunächst durch die Ideengewinnung generiert und anschließend über die Ideenselektion ausgewählt [19, S. 163], dazu werden Kreativitätstechniken genutzt [7, S. 15]. Kreativitätstechniken sind Denk- und Verhaltensregeln, um strukturiert Kreativität zu ermöglichen und so möglichst passende Ideen zur Problemlösung zu finden [8, S. 226].

\section{Methoden}

Als Kreativitätstechniken werden für die Ideengewinnung die 1-2-4-All- und für die Kategorisierung der Ideen die Evolution-Methode genutzt.

\section{1-2-4-All}

Bei der 1-2-4-All (vgl. Tab. 1), einer Methode der Liberating Structures, notieren die Teilnehmenden zunächst einzeln Ideen, um diese daraufhin mit Partner/innen zu teilen [11]. Anschließend besprechen sich jeweils 2 Paare und zuletzt stellt jede Vierergruppe eine Idee im Plenum vor [11]. Bei großen Plenargruppen kann nicht jede Vierergruppe eine Idee vorstellen, sodass eine Auswahl von insgesamt 3-4 Ideen erfolgt [11]. Bei dieser Auswahl der Ideen in Teilgruppen kann es dazu kommen, dass gute Ideen verloren gehen, wenn diese von stillen Teilnehmer/innen nicht vorgestellt werden. Da es aber bei der 1-2-4-All darum geht, alle einzubeziehen, wird in dieser Arbeit eine Vorauswahl der Ideen in den Phasen vor dem All ausgeschlossen. Die 1-2-4-All wird genutzt, um viele Personen gleichzeitig einzubinden und so Fragen, Ideen und Vorschläge zu generieren [11].

Für die Sammlung der Ideen wurde diese Methode ausgewählt, da sie laut Google Trends [9] zunehmend Beachtung findet. Weiterhin werden bei der 1-2-4-All-Methode die Ideen in jedem Schritt vorgestellt und überarbeitet, was wiederum die von Briggs und Reinig [2, S. 141] empfohlene Qualität der Ideen verbessert. Außerdem wird in den Schritten einer kognitiven Überlastung der Teilnehmenden vorgebeugt [20, S. 943], weil diese nicht mit jeder Idee einzeln konfrontiert werden. Außerdem haben sich die Expert/ innen aus den Interviews für die 1-2-4-All-Methode aus- 
Tab. 1 Übersicht 1-2-4-All [11]

\begin{tabular}{llll}
\hline Schritte & Name & Zeit & Beschreibung \\
\hline 1 & 1 & $1 \mathrm{~min}$ & Alle Teilnehmenden notieren ihre Ideen \\
2 & 2 & $2 \mathrm{~min}$ & Vorstellung und Weiterentwicklung der Ideen im Zweierteam \\
3 & 4 & $4 \mathrm{~min}$ & $\begin{array}{l}\text { Vorstellung und Weiterentwicklung der Ideen, indem sich 2 Paare zusammenfin- } \\
\text { den }\end{array}$ \\
4 & All & 5 min pro Vierergruppe & Vorstellung einer oder mehrerer Ideen pro Vierergruppe \\
\hline
\end{tabular}

Tab. 2 Anforderungen Expert/innen

\begin{tabular}{lll}
\hline Anforderungen & Expert/innen & \multicolumn{1}{c}{ Quelle } \\
\hline Die 1-2-4-All-Methode soll für den Prozess genutzt werden & $\begin{array}{l}\text { E1, E2, E3, E4, E6, E7, E9 } \\
\text { und E10 }\end{array}$ & [12, S. 193] \\
$\begin{array}{lll}\text { Es gibt in der 1-2-4-All-Methode Anpassungspotenzial in Form eines Zwischenschritts, der } \\
\text { die Anzahl der Ideen reduzieren soll }\end{array}$ & $\begin{array}{l}\text { E1, E7 und E8 } \\
\text { Für den Zwischenschritt wird eine Kategorisierung sowie eine Bewertung empfohlen }\end{array}$ & $\begin{array}{l}\text { E1, E3, E4, E5, E6, E7 und } \\
\text { [12, S. 195] }\end{array}$ \\
\hline
\end{tabular}

gesprochen, weil alle gehört und beteiligt werden und so bessere Ideen entstehen können.

\section{Evolution}

Für die Kategorisierung der Ideen, wurde die EvolutionMethode nach Briggs und Vreede [3] ausgewählt, die einerseits eine große Gruppe von Teilnehmenden zulässt und darüber hinaus die iterative Entwicklung der Kategorien ermöglicht. Die Evolution-Methode nimmt als Input eine Liste von nichtkategorisierten unsortierten Ideen auf und gibt nach der Durchführung als Ergebnis sowohl Kategorienamen als auch die Zuordnung der Ideen zurück [3, S. 95]. Sie eignet sich besonders gut, wenn die Kategorienamen vorab nicht offensichtlich sind [3, S. 95]. Um die Ideen zu kategorisieren, wird eine Idee zurzeit aus der Liste in der Gruppe diskutiert und eingeordnet [3, S. 95]. Über die „Evolution“ der Kategorien werden die Kategorienamen während des Clusterns gefunden und stetig verbessert [3, S. 95].

Normalerweise kostet dieses thinkLet viel Zeit, weil jede Idee einzeln in einer großen Gruppe diskutiert wird. Um hingegen viele Teilnehmer/innen einzubinden und die Zeit zu reduzieren, wird die Vorgehensweise in diesem Prozess angepasst. Alle erhalten im digitalen Tool einzelne Ideen nacheinander und ordnen diese einer passenden Kategorie zu. Die Kategorien sind allen gleichzeitig zugänglich und können angelegt und geändert werden. Durch diese Methodenauslegung arbeiten die Teilnehmenden zur gleichen Zeit auf ein gemeinsames Ergebnis hin.

\section{Forschungsmethodik}

Um die Forschungsfragen zu beantworten, wurden zunächst Experteninterviews nach Meuser und Nagel [15] durchge- führt und aufgenommen. Um danach strukturiert Erkenntnisse für die Lösung zu gewinnen, wurden die Aufnahmen transkribiert und nach Mayring [14] analysiert. Im Anschluss wurden Anforderungen abgeleitet, die die Grundlage für die Gestaltung des Ideenfindungsprozesses bilden.

Um den Ideenfindungsprozess in der Praxis zu evaluieren, wurden Workshops und Umfragen mit Teilnehmenden im Kontext der Genossenschaft nurdemteam eG durchgeführt.

Aus dem Workshop 1 stellte sich heraus, dass das Kategorisieren mit Standardsoftware nicht durchführbar ist. Daher wurde zusätzlich eine selbstgeschriebene Softwarelösung iterativ nach dem „Rapid Prototyping“ nach Schuh [23, S. 217] unter Einbeziehung der Expert/innen und Teilnehmenden entwickelt und in Workshop 2 evaluiert.

\section{Experteninterviews: Durchführung und Auswertung}

Neben der Literaturrecherche wurde im Rahmen der Anforderungsanalyse für den zu entwickelnden Prozess Experteninterviews durchgeführt. Damit die Daten aus den Interviews auch wertvoll und aussagekräftig sind, wurden als Expert/innen erfahrene Moderator/innen ausgewählt. Moderator/innen sind neutrale Personen, die eine Gruppe zielorientiert und methodisch zu einem Gruppenziel führen [22, S. 168]. Vorteilhaft sind an dieser Stelle Expert/innen, die Erfahrungen mit der 1-2-4-All-Methode sowie mit vielen Teilnehmenden haben. Letztendlich wurden 10 Expert/ innen im Umfang von circa 30 min befragt. Die Ergebnisse spiegeln sich in Workshop 1 sowie den Anforderungen für enEvo wider.

Mit den Anforderungen aus den Interviews wurde die erste Version des Ideenfindungsprozesses entwickelt (vgl. Tab. 2). 


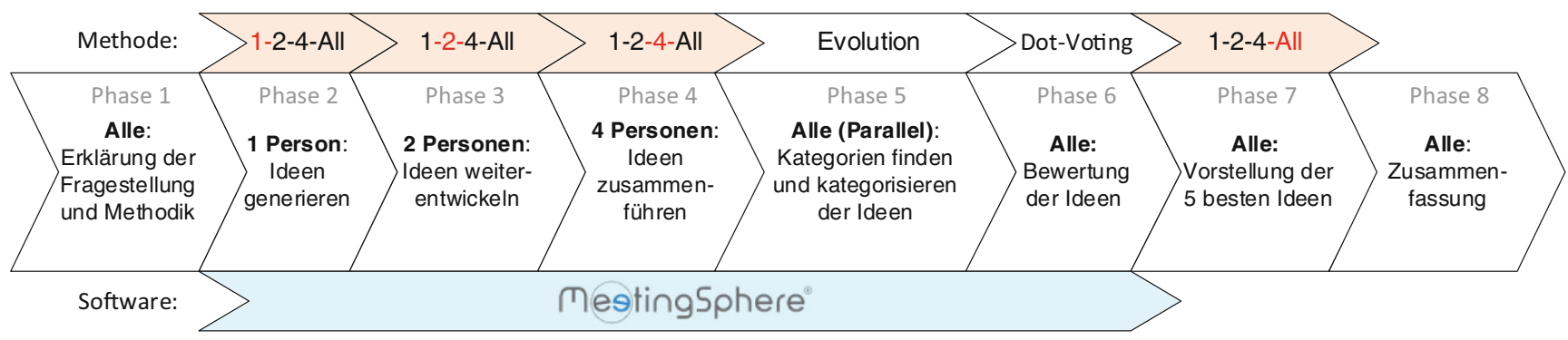

Abb. 1 Ideenfindungsprozess

\section{Entwurf des Ideenfindungsprozesses}

Der Ideenfindungsprozess ist ein systematisches Vorgehen zur Zusammenarbeit, der auch Kollaborationsprozess genannt wird. Dargestellt wird dieser Prozess in der Abb. 1. Entworfen wurde der Prozess nach dem Collaboration Engineering nach Leimeister [10], sodass wiederkehrende Kollaborationsprozesse mit hohem Wert entworfen werden können [4, S. 1]. Mit der systematischen Entwicklung soll der Prozess mit gleichbleibender Qualität wiederholbar sein und die Gruppenziele erreichen, ohne dass kostspielige erfahrene Moderator/innen engagiert werden müssen [4, S. 1 ff., 26, S. 140].

Der Prozess und dabei speziell die Phase 5 wurde nach den Anforderungen, die durch Interviews mit ausgewählten Expert/innen gewonnen wurden, entwickelt. Als Expert/innen wurden erfahrene Moderator/innen ausgewählt, die im Ideal auch Erfahrung mit der 1-2-4-All sowie groBen Personengruppen haben. Aus Platzgründen wird in diesem Artikel auf die Anforderungsanalyse verzichtet; sie ist nachzulesen in Makkai [12, S. $31 \mathrm{ff}$.].

Auf Grundlage der Erkenntnisse aus den Experteninterviews wurde der Ideenfindungsprozess konzipiert. Die, in diesem Prozess verwendete, 1-2-4-All-Methode kann nur bis zum 3. Schritt 1-2-4-All mit beliebig vielen Teilnehmenden durchgeführt werden. Ab dem 4. Schritt 1-2-4-All sind zu viele Ideen vorhanden. Um die Top-Ideen unter den Ideen zu finden und diese in gegebener Zeit zu präsentieren, muss eine Auswahl der Ideen getroffen werden. Die Auswahl erfolgt durch eine Kategorisierung und durch eine anschließende Bewertung der Ideen durch alle Teilnehmenden [21, S. 119]. Um die Auswahl der Ideen möglichst effizient zu gestalten, wird eine transparente Kategorisierung zur Sichtbarmachung von Mehrfachnennungen von Ideen verwendet [21, S. 119]. Außerdem wird, um dem Problem der skalierten Anzahl von Teilnehmenden gerecht zu werden, zwischen dem 3. Schritt 1-2-4-All und dem 4. Schritt 1-2-4-All diese Kategorisierung und Bewertung der Ideen eingebaut. Durch die Bewertung und Selektion der Top 5 wird die Menge von vielen Ideen auf die priorisierten 5 reduziert, sodass im 4. Schritt eine Vorstellung bei begrenzter Zeit erfolgen kann.
In der Abb. 1 wird der Ideenfindungsprozess visualisiert. Hierzu erfolgt zu Beginn des Prozesses in Phase 1 zunächst eine Erklärung der Fragestellung und der Methodik, zu denen die Teilnehmenden Fragen stellen können. Im Anschluss werden in der zweiten Phase die Ideen generiert. Die Generierung und die anschließende Weiterentwicklung in der dritten und vierten Phase des Prozesses erfolgt in MeetingSphere. In der Phase 5 werden die Ideen nach der Evolution-Methode gruppiert. In Phase 6 erfolgt eine Bewertung der kategorisierten Ideen. Darauf folgen die Vorstellung der Top-5-Ideen in Phase 7 im Plenum sowie ein Abschluss durch den Moderator in der letzten Phase.

\section{Validierung Ideenfindungsprozess: Workshop 1}

Um den entwickelten Ideenfindungsprozess zu untersuchen, wurde ein Workshop gemäß dem Ablauf (vgl. Abb. 1) geplant. Als exemplarisches Workshopziel wurde festgelegt, dass das Kollegium der Genossenschaft in 90 min Ideen generieren, clustern und die Top 5 der Ideen priorisieren und vorstellen soll. Durchgeführt wurde der Workshop 1 mit der Softwareunterstützung MeetingSphere, welches ein Software-Tool zur Kollaboration von vielen Teilnehmenden ist. Der Prozess wurde zeitgleich und an einem gemeinsamen Ort mit 19 Mitgliedern der eG veranstaltet. Anschließend wurden die Teilnehmenden in Form einer Online-Umfrage zu dem Workshop befragt.

\section{Erkenntnisse}

Nach der Durchführung des Workshops und einer Umfrage hat sich ein Problem offenbart. Das zeitgleiche Kategorisieren vieler Ideen (Phase 5, siehe Abb. 1) wurde seitens MeetingSphere nicht ausreichend unterstützt. Eine Recherche und ausführliche Begutachtung von Standardsoftware haben ergeben, dass es zu diesem Zeitpunkt keine andere Software für das manuelle zeitgleiche Kategorisieren von Ideen gibt. Um den Teilnehmenden das zeitgleiche Kategorisieren auf der Grundlage der Evolution-Methode zu ermöglichen, wurde eine Eigenentwicklung geplant. Nach 


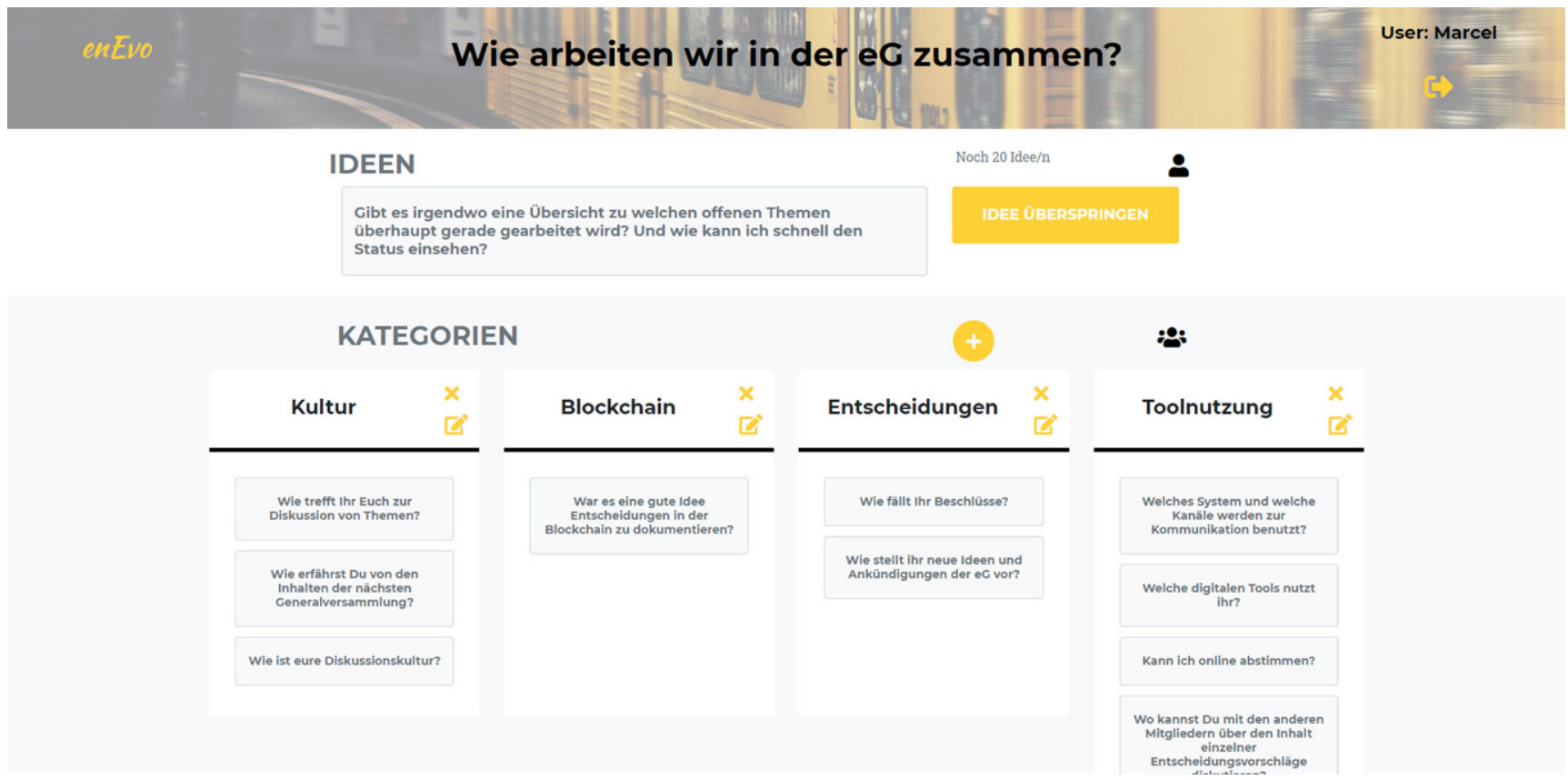

Abb. 2 Clusteransicht eines Nutzers

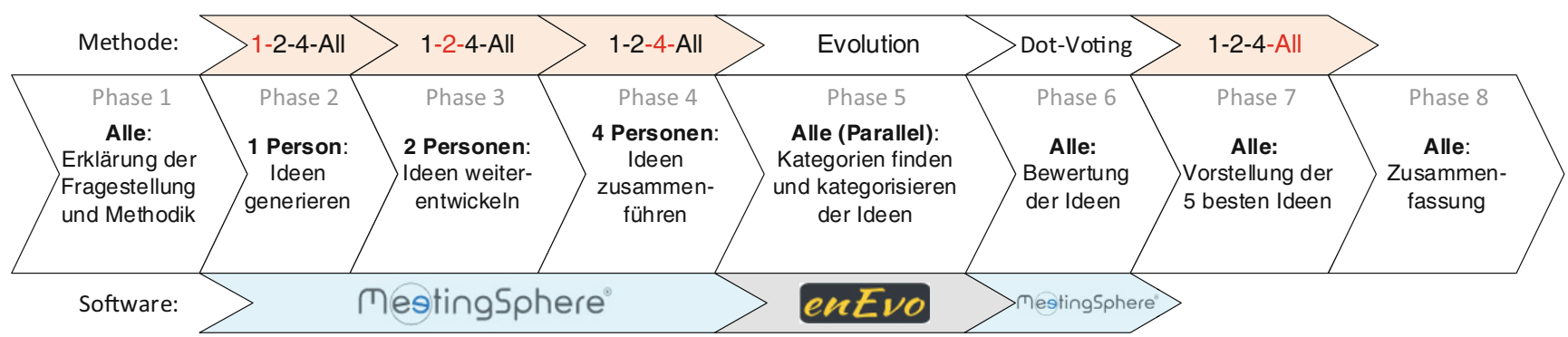

Abb. 3 Ideenfindungsprozess mit enEvo

den Anforderungen aus den Experteninterviews wurde die Anwendung implementiert.

Um die Software enEvo in Zusammenarbeit mit MeetingSphere im Ideenfindungsprozess zu evaluieren, wurde ein zweiter Workshop geplant.

\section{Prototyp enEvo}

Um das softwaregestützte Clustering (Phase 5, siehe Abb. 1) mit den Teilnehmenden durchzuführen, wird in diesem Abschnitt die eigenentwickelte Webanwendung enEvo (,Ideen Evolution“) vorgestellt. Innerhalb von enEvo können die Nutzer/innen Kategorien anlegen und Ideen zuordnen. Die Funktionsweise von enEvo richtet sich nach der Evolution Methode.

Technologisch baut die Webanwendung auf Frameworks der Webentwicklung auf. Verwendet wird als Backend Framework Spring Boot. Hiermit wird in Java die Backend-Funktionalität programmiert. Mit Thymeleaf sollen die Daten aus der H2-In-memory-Datenbank im HTML eingebettet und anschließend an den Browser versendet werden. Im Browser sollen durch Javascript Websockets implementiert werden, sodass die Daten unter den Clients eventbasiert ausgetauscht werden können.

In enEvo gibt es zwei verschiedene Ansichten, die der Moderation und die der Teilnehmenden. Im Folgenden wird der zeitliche Ablauf von Phase 5 in enEvo beschrieben, dieser gliedert sich in die drei Abschnitte Vorbereitung, Durchführung und Nachbereitung.

\section{Vorbereitung in enEvo}

Die Moderation importiert die gesammelten Ideen aus MeetingSphere und benennt das Thema der Clustering-Session. Währenddessen können die Teilnehmenden mit einem Namen beitreten. Anschließend ordnet die Moderation per $\mathrm{Zu}$ fallsalgorithmus die Ideen den Nutzer/innen zu. 


\section{Durchführung der Kategorisierung}

Gemäß der Evolution-Methode sollen sich die Teilnehmenden auf eine Idee zurzeit fokussieren. Dazu werden allen Teilnehmenden einzelne Ideen zugelost, die diese im Anschluss in Kategorien einsortieren. Die Kategorien werden dafür von den Nutzer/innen individuell angelegt und können im Nachhinein umbenannt werden. Das iterative Überarbeiten der Kategorienamen und der Ideenzuordnung ist vorgesehen und erwünscht. Nach dem Zuordnen oder Überspringen einer Idee werden den Teilnehmenden weitere einzelne Ideen zur Bearbeitung gegeben, bis ihr Ideenpool abgearbeitet ist. Übersprungene Ideen werden erneut in den Ideenpool eingespeist, bis eine Zuordnung zu einer Kategorie erfolgt. Wenn sich die Zuordnung der Ideen sowie die Kategorienamen nicht mehr verändern, ist das Clustering abgeschlossen. Ein Beispiel während des Clusterns in enEvo ist in Abb. $2 \mathrm{zu}$ sehen. In dieser gibt es drei Bereiche oben: Workshopthematik, mitte: persönlicher Ideenbereich, unten: gemeinsamer Kategorienbereich zum Clustern. Die Teilnehmenden verschieben die Ideen aus ihrem persönlichen Ideenbereich in eine Kategorie im gemeinschaftlichen Bereich.

\section{Nachbereitung}

Die Moderation exportiert die in Kategorien geclusterten Ideen für den Import in MeetingSphere und setzt den Workshop mit der Phase 6 Bewertung fort.

\section{Validierung enEvo: Workshop 2}

Um die Erkenntnisse aus Workshop 1 zu untersuchen, wurde ein zweiter Workshop gemäß dem Ablauf (vgl. Abb. 3) geplant. Dieser unterscheidet sich in der Softwareunterstützung in Phase 5. In dieser Phase wurde der zuvor vorgestellte Prototyp enEvo getestet. Der weitere Ablauf u. a. mit MeetingSphere und das gesetzte Workshopziel unterscheiden sich nicht zum Workshop 1. An diesem Workshop haben 9 Probanden teilgenommen. Anschließend wurden die Teilnehmenden wieder in Form einer Online-Umfrage zu dem Workshop befragt.

\section{Erkenntnisse}

Aus der Umfrage und dem Feedback aus dem Workshop 2 lässt sich ableiten, dass der Prozess im zweiten Durchlauf mit enEvo gut durchführbar war. Es konnte beobachtet werden, dass der Ablauf mit enEvo in der Phase 5 besser funktioniert hat. Jedoch wurde die Evolution-Methode mit ausreichend ( 3,89 von 6 Punkten) bewertet. Laut Umfrage wurde der Einsatz von enEvo mit 4,78 von 6 Punkten bewertet.
Dadurch war das allgemeine Feedback der Teilnehmenden auch insgesamt besser, der Workshop wurde insgesamt mit 5 von 6 Punkte bewertet.

Als hervorzuhebendes Feedback wurde von den Teilnehmenden eine Grenze der kognitiven Fähigkeiten angemerkt. Die Teilnehmenden wurden entlastet, da sie gemäß der Evolution-Methode nur eine Idee zurzeit clustern. Allerdings teilen sich die Teilnehmenden in der Webanwendung bei den Kategorien einen gemeinsamen Bereich. Daraus resultiert die Feststellung, dass aufgrund der hohen parallelen Bearbeitung der Ideen, vor allem während des Clusterns, sehr viele Eindrücke auf die Teilnehmenden einwirken und damit die menschlichen Kapazitäten überlastet werden könnten. Das liegt daran, dass alle Änderungen von allen Teilnehmenden im gemeinsamen Bereich auflaufen, z. B. das Karten-Verschieben oder das KategoriennamenVerändern.

Das Problem konnte gehändelt werden, da die Workshops gleichzeitig und gleichenorts stattfanden. Dadurch konnten sich die Teilnehmenden bei Verständnisproblemen untereinander austauschen und so der kognitiven Überlastung entgegenwirken. Eine weitere Möglichkeit ist, dass die Teilnehmenden nicht einzeln clustern, sondern in Kleingruppen. Dadurch finden in den Kleingruppen Diskussionen statt und nur eine Person bearbeitet anschließend den gemeinsamen Raum, sodass weniger Änderungen stattfinden.

\section{Diskussion und Fazit}

Im Rahmen der Validierung wurden 2 Workshops und Umfragen entwickelt und mit Probanden durchgeführt. Der erste wurde mit der bestehenden Software MeetingSphere ausgeführt, in dem festgestellt wurde, dass das synchrone Clustern mit vielen Teilnehmer/innen nicht ausreichend unterstützt wird. Der zweite Workshop wurde daher mit einer Kombination aus MeetingSphere und zum Clustern enEvo durchgeführt. Hierbei wurde untersucht, ob die Durchführung mit der eigenentwickelten Webanwendung enEvo im Kollaborationsprozess funktioniert und insgesamt bessere Ergebnisse liefert.

Um FF2, „Wie kann das manuelle Clustern im Ideenfindungsprozess durch Software unterstützt werden?“ beantworten zu können, wurde enEvo auf Grundlage der Evolution-Methode entwickelt. Der Ideenfindungsprozess mit enEvo wurde im Workshop 2 untersucht. Es konnte festgestellt werden, dass enEvo seine Zielvorgabe und die funktionalen Anforderungen erfüllt und die Testnutzer/innen die Software mit gut bewertet haben (4,78 von 6 Punkten).

FF1: ,Wie kann auf der Grundlage der 1-2-4-All-Methode ein Ideenfindungsprozess erstellt werden, der die digitale Ideenfindung im skalierten Teilnehmerfeld unterstützt?“ Aus den Evaluationen und Zyklen der Untersuchung hat 
sich gezeigt, dass der entworfene Prozess auf Grundlage der 1-2-4-All im skalierten Teilnehmendenfeld durch den Zwischenschritt des Clusterings und der Bewertung grundsätzlich funktioniert.

Der entwickelte Prozess erfüllt aus der Sicht der Moderation das zuvor festgelegte Ziel, dass das Kollegium der Genossenschaft in $60 \mathrm{~min}$ Ideen generiert, clustert und die Top 5 der Ideen priorisiert und vorstellt.

Die Umfrageergebnisse verdeutlichen, dass das Kollegium gegenüber der Skalierbarkeit der Methodik zuversichtlich ist. Es zeigt sich jedoch auch, dass die Meinungen der Teilnehmenden gespalten sind und manche aufgrund der Anzahl an Ideen und Duplikaten an der Skalierbarkeit zweifeln. Die Mehrheit vertritt allerdings die Meinung, dass sich der Prozess bis zu 60 Teilnehmende skalieren lässt, solange die Kommunikation untereinander gewährleistet ist. Sollte die Anzahl der Teilnehmenden 60 Personen überschreiten, ist zu bedenken, dass durch die mögliche Vielzahl an Idee-Duplikaten die Überlastung der kognitiven Kapazitäten der Teilnehmenden noch wahrscheinlicher wird. Diese entsteht dadurch, dass beim Clustering mehr Ideen vorhanden sind und dadurch bei vielen Teilnehmenden mehr Aktionen gleichzeitig stattfinden.

Bei der digitalen Durchführung des Prozesses hat sich herausgestellt, dass der Einsatz von MeetingSphere neben enEvo dafür gut geeignet ist, weil die Teilnehmenden dieses als gut bewertet haben und es vom Großteil intuitiv bedienbar war. Einige Benutzer/innen haben sich trotzdem eine Einführung gewünscht. Aus Moderationssicht wurde kein besseres Tool für diesen Ideenfindungsprozess gefunden.

Eine analoge Durchführung des Workshops ist zu bevorzugen, wenn eine minimale Hürde für den digitalen Zugang vorhanden sein sollte. Generell ist der Workshop am gleichen Ort und gleichzeitig zu bevorzugen, weil die persönliche Kommunikation nicht durch digitale Mittel zu ersetzen ist. Die digitalen Geräte, wie beispielsweise ein Laptop, sind im digitalen Workshop vor Ort eher ein Hilfsmittel als eine Notwendigkeit. Eine Stimme unter den Expert/ innen sagte: „Also tatsächlich ist die analoge Technik die schnellste, die existiert. Nichts ist einfacher als vor Ort, alle an einem Ort und das analog durchzuführen." $[12$, S. 156]. Wenn die Tools daher nicht maßgeblich zur Unterstützung beitragen oder notwendig sind, sollte ein analoger Workshop bevorzugt werden. Ein Nachteil bei einem digitalen Workshop sind mögliche technische Probleme, die bei analogen Workshops nicht vorhanden sind. Der digitale Workshop ist dem analogen vorzuziehen, wenn Vorteile, wie beispielswiese das gemeinsame Arbeiten an einem Clustering, vorliegen.

Allgemeingültig lässt sich zusammenfassen, dass die Ergebnisse dieser Arbeit an einem exemplarischen Prozess der Genossenschaft gezeigt wurden und es anzunehmen ist, dass der Prozess auch in anderen Kontexten und Themen- bereichen verwendet werden kann. Der Beweis für diese Annahme ist allerdings noch ausstehend und nicht Teil des vorliegenden Artikels. Zusätzlich ist anzumerken, dass für eine hinreichende Aussagekraft weitere Workshops mit einer höheren Personenanzahl notwendig sind.

\section{Schlusswort}

In den Testworkshops sowie Evaluierungen hat sich der Ideenfindungsprozess mit den digitalen Tools stetig verbessert und nach mehreren Durchläufen bewährt. Insgesamt kann mit dem entwickelten Prozess, der digital und in Verbindung mit MeetingSphere und der Webanwendung enEvo durchgeführt wird, das Problem der wachsenden Anzahl von Teilnehmenden besser bewältigt werden. Es ist allerdings zu bedenken, dass die Menschen eine geistige Kapazitätsgrenze besitzen und im digitalen Workshop nicht durch zu viele Einflüsse überlastet werden sollten.

Durch immer mehr digital ausgeführte Workshops gewinnen digitale Tools für die Gestaltung und Moderation an Bedeutung. Es konnten im Rahmen der Workshops bereits einige Ideen für die Genossenschaft generiert werden. In Zukunft werden sicher noch weitere Workshops der Genossenschaft mit dem geschaffenen Kollaborationsprozess ausgeführt. Das Bestreben nach digital unterstützten Workshops vor Ort unter Anwendung eines manuellen softwaregestützten Clusterings ist ein Beginn in dieser Entwicklung, die sich in den nächsten Jahren fortsetzen kann.

Funding Open Access funding enabled and organized by Projekt DEAL.

Open Access Dieser Artikel wird unter der Creative Commons Namensnennung 4.0 International Lizenz veröffentlicht, welche die Nutzung, Vervielfältigung, Bearbeitung, Verbreitung und Wiedergabe in jeglichem Medium und Format erlaubt, sofern Sie den/die ursprünglichen Autor(en) und die Quelle ordnungsgemäß nennen, einen Link zur Creative Commons Lizenz beifügen und angeben, ob Änderungen vorgenommen wurden.

Die in diesem Artikel enthaltenen Bilder und sonstiges Drittmaterial unterliegen ebenfalls der genannten Creative Commons Lizenz, sofern sich aus der Abbildungslegende nichts anderes ergibt. Sofern das betreffende Material nicht unter der genannten Creative Commons Lizenz steht und die betreffende Handlung nicht nach gesetzlichen Vorschriften erlaubt ist, ist für die oben aufgeführten Weiterverwendungen des Materials die Einwilligung des jeweiligen Rechteinhabers einzuholen.

Weitere Details zur Lizenz entnehmen Sie bitte der Lizenzinformation auf http://creativecommons.org/licenses/by/4.0/deed.de.

\section{Literatur}

1. Bahnprojekt Stuttgart-Ulm (2019) Chronologie. http://www.bahn projekt-stuttgart-ulm.de/projekt/historie/chronologie/. Zugegriffen: 28. Febr. 2021 
2. Briggs RO, Reinig BA (2010) Bounded ideation theory. J Manag Inf Syst 27(1):123-144. https://doi.org/10.2753/MIS07421222270106

3. Briggs R, de Vreede GJ (2009) Thinklets: building blocks for concerted collaboration

4. Briggs R, de Vreede GJ, Kolfschoten G, Dean DL (2006) Defining key concepts for collaboration engineering

5. Dialogforum Schiene Nord (2015) Ergebnis des Dialogforums. http://www.dialogforum-schiene-nord.de/ergebnis. Zugegriffen: 28. Febr. 2021

6. Gassmann O (2013) Innovation: Zufall oder Management? In: Gassmann O, Sutter P (Hrsg) Praxiswissen Innovationsmanagement. Von der Idee zum Markterfolg, Bd. 3. Hanser, München, S $1-23$

7. Gawlak M (2014) Kreativitätstechniken im Innovationsprozess. Von den klassischen Kreativitätstechniken hin zu webbasierten kreativen Netzwerken. Diplomica, Hamburg

8. Geschka H (2006) Kreativitätstechniken und Methoden der Ideenbewertung. In: Sommerlatte T, Beyer G (Hrsg) Innovationskultur und Ideenmanagement. Strategien und praktische Ansätze für mehr Wachstum, 1. Aufl. Symposion, Düsseldorf, S 217-250 (https://books.google.de/books?id=BvPEk-wyTNsC\& printsec $=$ frontcover $\& \mathrm{hl}=\mathrm{de} \# \mathrm{v}=$ onepage $\& \mathrm{q} \& \mathrm{f}=$ false, zuletzt geprüft am 28.02.2021)

9. Google Trends (2019) Googleaufrufe liberating structures und 1-24-all. https://trends.google.de/trends/explore?date=all\&q=liberating \%20structures. Zugegriffen: 18. Apr. 2019

10. Leimeister JM (2014) Collaboration engineering. Springer, Berlin, Heidelberg

11. Lipmanowicz H, McCandless K Liberating structures-1-2-4-all. http://www.liberatingstructures.com/1-1-2-4-all/. Zugegriffen: 28. Febr. 2021

12. Makkai M (2019) Digitale Ideenfindung im skalierten Teilnehmerfeld. Uni Hamburg, Hamburg

13. Mast C (2014) Interne Unternehmenskommunikation: Mitarbeiter führen und motivieren. In: Zerfaß A, Piwinger M (Hrsg) Handbuch Unternehmenskommunikation. Strategie - Management - Wertschöpfung, 2. Aufl. Bd. 32. Springer, Wiesbaden, S 1121-1140

14. Mayring P, Fenzl T (2014) Qualitative Inhaltsanalyse. In: Nina B, Jörg B (Hrsg) Handbuch Methoden der empirischen Sozialforschung, Bd. 3. Springer VS, Wiesbaden, S 543-556
15. Meuser M, Nagel U (2009) Das Experteninterview - konzeptionelle Grundlagen und methodische Anlage. In: Susanne P (Hrsg) Methoden der vergleichenden Politik- und Sozialwissenschaft. VS, Wiesbaden, S 465-479

16. Mirow M (2003) Wertsteigerung durch Innovation. In: Max JR, Herbert AH, Michael M (Hrsg) Perspektiven der Strategischen Unternehmensführung. Gabler, Wiesbaden, S 331-346

17. Osborn AF (1953) Applied imagination: principles and procedures of creative thinking: Scribners

18. Paulus PB, Kohn NW, Arditti LE (2011) Effects of quantity and quality instructions on brainstorming. J Creat Behav 45(1):38-46

19. Pieper R (1992) Innovation. In: Rüdiger P (Hrsg) Lexikon Management. Gabler, Wiesbaden, S 163-188

20. Reinig BA, Briggs RO (2013) Putting quality first in ideation research. Group Decis Negot 22(5):943-973

21. Schawel C, Billing F (2014) Ideenbewertung. In: Christian S, Fabian B (Hrsg) Top 100 Management Tools, 5. Aufl. Gabler, Wiesbaden, S 119-121

22. Schawel C, Billing F (2014) Moderation. In: Christian S, Fabian B (Hrsg) Top 100 Management Tools, 5. Aufl. Gabler, Wiesbaden, S $168-170$

23. Schuh G (2011) Handbuch Produktion und Management, 2. Aufl. Springer Vieweg, Berlin

24. Stern T, Jaberg H (2010) Erfolgreiches Innovationsmanagement. Erfolgsfaktoren - Grundmuster - Fallbeispiele, 4. Aufl. Gabler, Wiesbaden

25. Voigt K-I (2008) Industrielles Management. Industriebetriebslehre aus prozessorientierter Sicht. Springer, Berlin, Heidelberg (Springer-Lehrbuch)

26. de Vreede GJ, Briggs R, Kolfschoten G (2006) Thinklets: a pattern language for facilitated and practitioner-guided collaboration processes. Int J Comput Appl Technol 25:140-154

27. Waibel R (2015) Die 7 Prinzipien zum Unternehmenserfolg. Einfach, zukunftsweisend, praxisorientiert, 1. Aufl. Hanser, München

28. Weimar J (2010) Moderation und Grossgruppenbegleitung. Bildungsmaterial. MediaService, Berlin 